\title{
Computer simulation of Thermal Focal Length of Laser Crystal and Thermally-Near-Unstable Resonator
}

\author{
Geng Aicong ${ }^{1,3}$, Li Baohe ${ }^{1}$, Chen Xiaobai ${ }^{1}$ \\ Department of Physics \\ Beijing Technology and Business University \\ Beijing 100048, China
}

\author{
Yang Xiaodong ${ }^{2}$ \\ Department of Physics \\ Jiaying College \\ Meizhou, 515015 China
}

\author{
Yang Mingjiang ${ }^{3}$ \\ Institute of mechanics \\ Chinese Academy of Science \\ Beijing 100190, China
}

\begin{abstract}
A simple method determining the thermal focal length of gain medium was investigated. Then by using computer simulation results, a novel configuration was proposed. In the new configuration, the gain medium acts as a mode limiter at maximal pump power, which restrains resonance of high-order modes and guarantees not only high output power but also high beam quality. An LD side-pumped Nd:YAG laser with output power of $400 \mathrm{~W}$ at $1064 \mathrm{~nm}$ was obtained experimentally with the new configuration. The experimental results are in agreement with theoretical predictions.
\end{abstract}

Keywords- laser; computer simulation;thermal focal length

\section{INTRODUCTION}

Scaling of the output power and brightness of diodepumped solid-state laser sources to meet the needs of ever demanding applications continues to preoccupy many within the laser community. The use of a multirod solid-state laser system is one of the most promising approaches to generating a high-power laser beam, at high beam quality, with a wide range of output power [1]. However, when the laser runs at high pump power, thermal lens of every rod induces variations of output beam parameters that could limit the design and operation of solid-state laser devices such as the stability of, width of lasing mode and the beam quality. So it is the effective means by measuring the thermal focal length of gain medium to investigate the thermal effect. General experimental methods of measuring thermal focal length include: probe beam method [2-4], interference detecting method [5], unstable-resonator method [6-7], and transverse mode beat frequency [8]. But experimental steps are usually complex and time-consuming.

In this paper, we propose a simple method determine the thermal focal length of gain medium. Firstly, we could measure the maximum laser resonator length by an asymmetric plat-plat cavity under a certain pump power and deduce the corresponding waist size of the laser with the same parameters. Basing on the theory of standard matrix transform, we can achieve the thermal focal length of gain medium by using of computer simulation. This method has advantages such as simple setup and easy operation. On basis of the analysis, a novel configuration was proposed. In the new configuration, the gain medium acts as a mode limiter at maximal pump power, which restrains resonance of high-order modes and guarantees not only high output power but also high beam quality. We experimentally gained $400 \mathrm{~W}$ output power at $1064 \mathrm{~nm}$ by an LD side-pumped Nd:YAG laser with the new configuration. The experimental results are in agreement with theoretical predictions.

\section{THEORETICAL ANALYSIS}

We begin by considering in more detail the adverse effects of heat generation within LD-pumped solid-state lasers. In general, a detailed knowledge of thermal lens can be quite difficult as it requires either accurate measurement of the thermally induced phase difference as a function of transverse position, or, if the values of all the required parameters are known, a numerical calculation of thermal lens taking into account the various contributing factors. Alternatively, if axial heat flow is neglected and consideration is limited to the contribution to thermal lens due to the temperature dependence of the refractive index, then an approximate expression for the thermal lens focal length can be gotten as the following[9]

$$
f=\frac{K A}{P_{d}}\left(\frac{1}{2} \frac{d n}{d T}+n_{0}^{3} \alpha C_{r, \phi}+\frac{\alpha r_{0}\left(n_{0}-1\right)}{L}\right)^{-1}
$$

where $\mathrm{A}$ is the rod cross-sectional area, $K$ is the thermal conductivity of the laser material, $P_{d}$ is the total heat dissipated in the $\operatorname{rod}, \mathrm{d} n / \mathrm{d} T$ is the change of refractive index with temperature, $\alpha$ is the thermal expansion coefficient, $C_{r}$ and $C_{\phi}$ are functions of the elasto-optical coefficients for radial and tangential component of the polarized light, $L$ is the sample length, $r_{0}$ is the radius of the laser rod, $n_{0}$ is the refractive index in the center of the rod.

It can be seen from the above formula that, laser rod thermal focal length is determined by many factors, which are difficult to obtain accurately. A simple theoretical calculation can not truly reflect the characteristics of thermal focal length. 
Therefore, by combining experimental method with computer simulation calculation, we accurately measured the thermal focal length simply and practically.

(a)

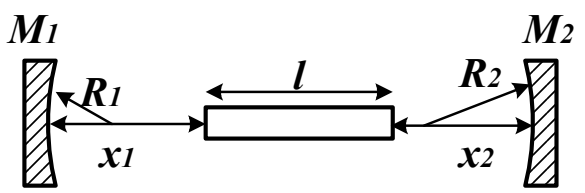

(b)

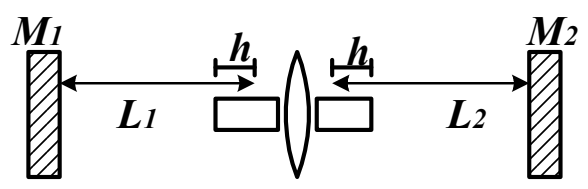

(c)

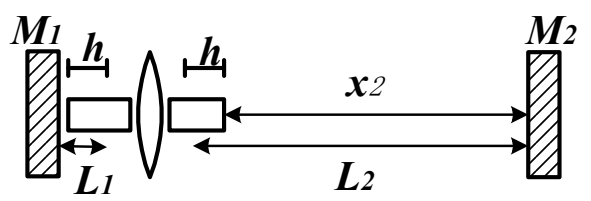

Figure 1. (a) Schematic representation of a simple laser cavity of length $x_{1}+$ $x_{2}+l$. The laser rod is of length $l$; (b) The laser rod is replaced by isotropic material and a thin lens, the cavity arms are measured from the principal plane, h.; (c) Ideal asymmetrical resonator where the laser rod is approximated by a thin lens.

The modeling analysis of this laser system is complicated because the transverse mode structure is dependent on the strength of thermal lens; to simplify analysis we make the assumption that the essential characteristics of the laser cavity (when approaching instability) can be modeled as the lowest order transverse mode. The stability of a laser resonator can be characterized by its equivalent g-parameters [10], where the rod can be approximated to first order by a thin spherical lens of focal length $f$, hence

$$
g_{1}=1-\frac{L_{2}}{f}-\frac{L^{\prime}}{R_{1}}
$$

and

$$
g_{2}=1-\frac{L_{1}}{f}-\frac{L^{\prime}}{R_{2}}
$$

where

$$
L^{\prime}=L_{1}+L_{2}-\frac{L_{1} L_{2}}{f}
$$

For the above equations, $f$ is the thermal focal length of gain medium, $R_{1,2}$ are the radius of curvature of the end mirrors and the lengths of the cavity arms are $L_{1,2}$ as shown in Fig. 1(a). $x_{1}$, ${ }_{2}$ are the distances between the mirror and the corresponding end of the rod. The laser rod (of length $l$ ) can be approximated by a thin lens plus a length of isotropic medium of refractive index $n_{0}$ with the principal planes $(h)$ of the lens defined from the ends of the laser rod. This is shown schematically in Fig. 1(b). At long focal lengths, it can be assumed the principle plane position (hence arm length) is independent of induced focal length, i.e.

$$
h=\frac{l}{2 n_{0}}
$$

Considering the length of the rod then

$$
L_{1}=x_{1}+h \quad L_{2}=x_{2}+h
$$

To simplify the analysis and experiment the cavity mirrors are chosen to be plane parallel. The cavity stability equation is

$$
0<g_{1} g_{2}=\left(1-\frac{L_{1}}{f}\right)\left(1-\frac{L_{2}}{f}\right)<1
$$

Hence the cavity stability of this simple resonator is dependent only on the focal length $(f)$ of the intra-cavity lens and the length of the cavity $\left(x_{1}+x_{2}+l\right)$.

In conventional design, the cavity is configured so that the two arms of the cavity are equal. At the critical rod focal length corresponding to $f=L_{1}=L_{2}$, the transverse laser modes are unstable. However, exact symmetrical cavity demands high precision and difficult to handle. Then we introduced asymmetrical cavity, $L_{1} \neq L_{2}$, as shown in Fig. 1(c). For increasing focal strength the asymmetrical cavity changes from $g_{1} \cdot g_{2}=1$ to $g_{1} \cdot g_{2}=0$ in a resonator stability diagram. At the critical rod focal length corresponding to $g_{1} \cdot g_{2}=0$, the transverse laser modes are unstable. The cavity stability equation, simplifies to

$$
f=L_{2}=x_{2}+\frac{l}{2 n_{0}}
$$

When measuring thermal focal lengths at different pump power, the position of the laser module and the cavity mirror $M_{1}$ can be fixed, and only the position of cavity mirror $M_{2}$ need to be change. Therefore, this method has advantages such as simple setup and easy operation.

\section{EXPERIMENTAL RESULTS}

On the basis of the above theory, the thermal focal length was measured accurately, and then a thermally-near-unstable resonator was proposed. By using $\mathrm{ABCD}$ ray propagation matrix as an auxiliary tool [11], the beam radius of fundamental mode on the Nd:YAG rod and the cavity mirror was computer simulated.

As shown in Fig.2, in this new resonator, when the laser works at maximal pump power, which is the power stable point, the laser is thermally-near-unstable. The laser beam size on the Nd:YAG rod is larger than that on the cavity mirror. Therefore, gain medium acts as a mode limiter at maximal pump power, which restrains resonance of high-order modes and guarantees not only high output power but also high beam quality. Finally, we experimentally gained $400 \mathrm{~W}$ output power 
at $1064 \mathrm{~nm}$ by an LD side-pumped Nd:YAG laser with the new configuration. The output power as a function of the LD pump current is showed in fig.3. The experimental results are in agreement with theoretical predictions.

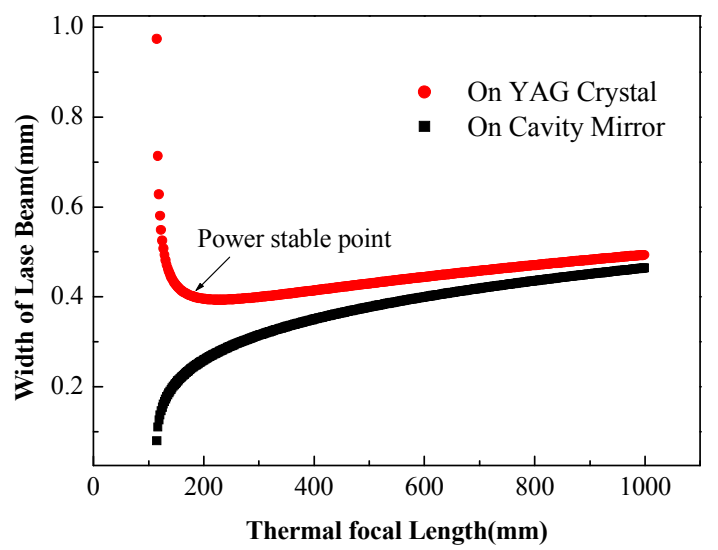

Figure 2. The beam radius of fundamental mode on the Nd:YAG rod and the cavity mirror at different thermal focal length

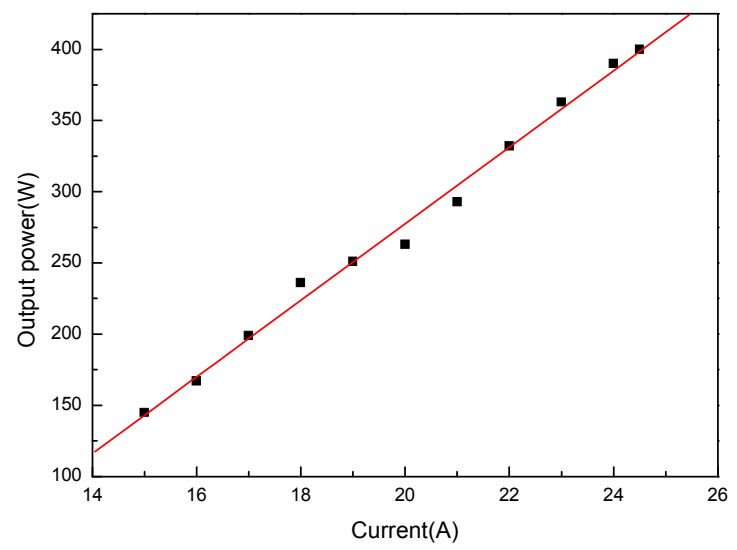

Figure 3. Experimental output power as a function of the LD pump current

\section{ACKNOWLEDGMENT}

This work was supported by Funding Project for Academic Human Resources Development in Institutions of Higher Learning Under the Jurisdiction of Beijing Municipality (PHR201007122), Funding Project for Innovation on Science, Technology and Graduate Education in Institutions of Higher Learning Under the Jurisdiction of Beijing Municipality, the National Natural Science Foundation of China (Grant No. 61007021 and 11075176) and the China Postdoctoral Science Foundation (Grant No.20080430562).

\section{REFERENCES}

[1] K. P. Drieger, R. M. Ifflander, and H. Weber, "Multi-rod resonators for high-power solid-state lasers with improved beam quality," IEEE J. Quantum Electron. Vol.24, pp.665-674, 1988.

[2] Koechner W, "Thermal Lensing in a Nd:YAG Laser Rod," Appl. Opt. vol.9, pp.2548-2553, 1970 .

[3] Kortz H P, Ifflander R and Weber H, "Stability and beam divergence of multimode lasers with internal variable lenses,"Appl. Opt. vol.20, pp.4124-4134,1981.

[4] Driedger K P, Krause W and Weber H, "Average refractive powers of an alexandrite laser rod,”Opt. Commun. vol.57, pp.403-406, 1986.

[5] Omatsu T, Kato Y, Shimosegawa M, Hasegawa A, Ogura I, "Thermal effects in laser diode pumped self-frequency-doubled $\mathrm{Nd}_{\mathrm{x}} \mathrm{Y}_{1^{-}}$ ${ }_{x} \mathrm{Al}_{3}\left(\mathrm{BO}_{3}\right)_{4}(\mathrm{NYAB})$ microchip laser," Opt . Commun.vol.118, pp.302$308,1995$.

[6] Liu Jun-hai, Lu Jianren, Lu Junhua, et al. "Thermal lens determination of end-pumped solid-state lasers by a direct approach", Chin Phys Lett., Vol.16(3), pp.181-183, 1999.

[7] D.G. Lancaster, J.M. Dawes, "Thermal-lens measurement of a quasi steady-state repetitively flash lamp-pumped $\mathrm{Cr}$, Tm, Ho:YAG laser", Opt. Laser Technol., Vol.30, pp.103-108, 1998.

[8] Ozygus B and Zhang Q C. "Thermal lens determination of end-pumped solid-state lasers using primary degeneration modes," Appl. Phys. Lett. vol.71, pp.2590-2592, 1997.

[9] Koechner W, Bass M, Solid-state Laser, New York:Springer-verlag, 2003, pp.257.

[10] Magin V., "Resonators for solid-state lasers with large-volume fundamental mode and high alignment stability", Appl. Opt., vol.25,pp. 107-117, 1986.

[11] B. D. Lü, Laser optics-laser beam propagation and beam quality control, Chengdu: Sichuan University Press, 1992.Chap.8. 